\title{
Uniqueness Results for Co-Circular Central Configurations for Power-Law Potentials
}

\author{
Josep M. Cors*
}

\author{
Glen R. Hall ${ }^{\dagger}$
}

May 2, 2014

\author{
Gareth E. Roberts ${ }^{\ddagger}$
}

\begin{abstract}
For a class of potential functions including those used for the planar $n$-body and $n$-vortex problems, we investigate co-circular central configurations whose center of mass coincides with the center of the circle containing the bodies. Useful equations are derived that completely describe the problem. Using a topological approach, it is shown that for any choice of positive masses (or circulations), if such a central configuration exists, then it is unique. It quickly follows that if the masses are all equal, then the only solution is the regular $n$-gon. For the planar $n$-vortex problem and any choice of the vorticities, we show that the only possible cocircular central configuration with center of vorticity at the center of the circle is the regular $n$-gon with equal vorticities.
\end{abstract}

Key Words: Central configuration, $n$-body problem, $n$-vortex problem, co-circular central configuration

\section{Introduction}

Central configurations are important solutions in the Newtonian $n$-body problem. They arise naturally when searching for homographic solutions, where the overall shape of the configuration is preserved throughout the motion. In the planar $n$-body problem, they help identify bifurcations in the topology of the integral manifolds since those changes occur only for energy levels where a central configuration is present [14]. They also play a significant role in astronomy and spacecraft mission design by providing useful information on nearby orbits, information that has been cleverly exploited by scientists to design inexpensive space missions [11].

This work focuses on those central configurations that lie on a common circle, a co-circular central configuration, with the additional property that the center of mass of the configuration coincides with the center of the circle. In [6], Chenciner asked if the only solution of this type was the regular $n$-gon with equal masses. The first to provide an answer to Chenciner's question

\footnotetext{
*Departament de Matemàtica Aplicada III, Universitat Politècnica de Catalunya, cors@epsem.upc.edu

$\dagger$ Dept. of Mathematics and Statistics, Boston University, rockford@bu.edu

${ }^{\ddagger}$ Dept. of Mathematics and Computer Science, College of the Holy Cross, groberts@holycross.edu
} 
was Hampton, who proved that the only four-body co-circular central configuration with center of mass coinciding with the center of the circle is the square with equal masses [8]. Llibre and Valls have announced that the regular pentagon (again with equal masses) is the only co-circular central configuration with this special property for $n=5$ [10]. This question is listed as Problem 12 in a collection of important open problems in celestial mechanics compiled by Albouy, Cabral and Santos [1].

In this paper we extend Chenciner's question to a range of potential functions that includes both the Newtonian case and the $n$-vortex problem. We derive two systems of equations that completely describe these special central configurations. Then, using a topological approach reminiscent of Moulton's [15], we show that for any fixed choice of masses, if there is a co-circular central configuration whose center of mass coincides with the center of the circle, it is unique. It follows quickly that if the masses are assumed to be equal, then the regular $n$-gon is the unique solution. In the case of $n$ vortices, our approach yields a complete solution to Chenciner's question: for any $n$, a co-circular central configuration whose center of vorticity coincides with the center of the circle must have equal strength vorticities, and is therefore the regular $n$-gon.

\section{Co-Circular Central Configurations}

We begin with the defining equations for a planar central configuration. Let $q_{i} \in \mathbb{R}^{2}$ and $m_{i}$ denote the position and mass, respectively, of the $i$-th body. We denote $r_{i j}=\left\|q_{i}-q_{j}\right\|$ as the distance between the $i$-th and $j$-th bodies. If $M=\sum_{i=1}^{n} m_{i}$ denotes the sum of the masses, then the center of mass is given by $c=\frac{1}{M} \sum_{i=1}^{n} m_{i} q_{i}$.

\subsection{The generalized Newtonian potential}

Consider a family of potential functions $U_{\alpha}$ of the form

$$
U_{\alpha}=\sum_{i<j} \frac{m_{i} m_{j}}{r_{i j}^{\alpha}}
$$

where $\alpha>0$ is a real parameter. The Newtonian $n$-body problem corresponds to the case $\alpha=1$. A planar central configuration is a special set of distinct positions $q_{i} \in \mathbb{R}^{2}$ satisfying

$$
\sum_{j \neq i}^{n} \frac{m_{i} m_{j}\left(q_{j}-q_{i}\right)}{r_{i j}^{\alpha+2}}+\frac{\lambda}{\alpha} m_{i} q_{i}=0 \quad \text { for each } i \in\{1, \ldots, n\}
$$

and for some scalar $\lambda$ independent of $i$. Given any planar central configuration, there is a corresponding periodic solution, called a relative equilibrium, for which the bodies rigidly rotate about the center of mass. Here we have assumed, without loss of generality, that the center of mass $c$ is at the origin. If $q=\left(q_{1}, \ldots, q_{n}\right)$ is a central configuration, then rotating each body about the origin by the same angle produces a new central configuration. We follow the standard practice of identifying configurations similar under rotational symmetry.

The system of equations defined by (1) can be written more compactly as

$$
\nabla U_{\alpha}(q)+\lambda \nabla I(q)=0
$$


where $I$ is one-half the moment of inertia, $I=\frac{1}{2} \sum_{i=1}^{n} m_{i}\left\|q_{i}\right\|^{2}$. Note that $I$ is a homogeneous function of degree 2 while $U_{\alpha}$ is homogeneous of degree $-\alpha$. Taking the dot product of equation (2) with $q$ then yields the useful formula

$$
\lambda \equiv \lambda(\alpha)=\frac{\alpha U_{\alpha}}{2 I}
$$

Since $\alpha>0$ and $m_{i}>0$, we must have $\lambda>0$. Assuming the equations of motion are in the standard form $m_{i} \ddot{q}_{i}=\partial U_{\alpha} / \partial q_{i}$, the angular velocity of the corresponding relative equilibrium is given by $\sqrt{\lambda}$. Summing all the equations in system (1) quickly gives $\sum_{i} m_{i} q_{i}=0$, which confirms that the center of mass of the central configuration is at the origin.

We call a planar central configuration co-circular if all the bodies lie on a common circle. We are interested in the co-circular central configurations whose center of mass coincides with the center of the circle. Toward that end, suppose we have a planar central configuration where all the bodies lie on the unit circle. Let $q_{i}=\left(\cos \theta_{i}, \sin \theta_{i}\right)$ where $\theta_{i} \in[0,2 \pi)$ for each $i$. The angles must satisfy, for each $i \in\{1, \ldots, n\}$,

$$
\sum_{j \neq i}^{n} \frac{m_{j}}{r_{i j}^{\alpha+2}}\left[\begin{array}{c}
\cos \theta_{j}-\cos \theta_{i} \\
\sin \theta_{j}-\sin \theta_{i}
\end{array}\right]+\frac{\lambda}{\alpha}\left[\begin{array}{c}
\cos \theta_{i} \\
\sin \theta_{i}
\end{array}\right]=0 .
$$

We will divide these equations into angular and radial components. The equations involving the angular components are obtained by computing the dot product of the $i$-th equation in system (4) with the vector $\left[-\sin \theta_{i}, \cos \theta_{i}\right]^{T}$. This yields the system

$$
\sum_{j \neq i}^{n} \frac{m_{j}}{r_{i j}^{\alpha+2}} \sin \left(\theta_{j}-\theta_{i}\right)=0, \quad \text { for each } i \in\{1, \ldots, n\} .
$$

If equation (5) holds for some $i$, then the force (due to gravity for the case $\alpha=1$ ) acting on the $i$-th body points toward the origin (the center of mass). Using the fact that

$$
r_{i j}=\sqrt{2-2 \cos \left(\theta_{j}-\theta_{i}\right)}=2 \sin \left(\frac{\left|\theta_{j}-\theta_{i}\right|}{2}\right),
$$

equation (5) simplifies to

$$
-\frac{1}{2^{\alpha+1}} \sum_{j \neq i}^{n} \frac{\delta_{i j} m_{j} \cos \left(\frac{\theta_{j}-\theta_{i}}{2}\right)}{\left[\sin \left(\frac{\left|\theta_{j}-\theta_{i}\right|}{2}\right)\right]^{\alpha+1}}=0, \quad \text { where } \delta_{i j}=\left\{\begin{array}{lc}
1 & \text { if } \theta_{i}-\theta_{j}>0 \\
-1 & \text { if } \theta_{i}-\theta_{j}<0 .
\end{array}\right.
$$

It is interesting to note that equation (7) can also be derived by using a variational approach. Using the principle of least action and beginning with a relative equilibrium solution on a circle with center of mass equivalent to the center of the circle, a perturbing path that only varies the angles leads to equation (7).

To derive the equations for the radial components, we take the dot product of the $i$-th equation in system (4) with the vector $\left[\cos \theta_{i}, \sin \theta_{i}\right]^{T}$. In this case, we obtain the system

$$
\sum_{j \neq i}^{n} \frac{m_{j}}{r_{i j}^{\alpha+2}}\left(\cos \left(\theta_{j}-\theta_{i}\right)-1\right)+\frac{\lambda}{\alpha}=0, \quad \text { for each } i \in\{1, \ldots, n\} .
$$


Using (6), equation (8) simplifies to

$$
\sum_{j \neq i}^{n} \frac{m_{j}}{r_{i j}^{\alpha}}=\frac{2 \lambda}{\alpha}, \quad \text { for each } i \in\{1, \ldots, n\} .
$$

If equation (9) holds for some $i$, then the magnitude of the force vector acting on the $i$-th body is the correct length to satisfy equation (1).

Equations (7) and (9) define a system of $2 n$ equations that are both necessary and sufficient for a central configuration on the unit circle to have its center of mass at the origin.

Proposition 2.1. Suppose that $q$ is a configuration on the unit circle with masses $m_{1}, \ldots, m_{n}$, whose center of mass is at the origin. Then $q$ is central if and only if its angles and masses satisfy both equation (7) and (9) for each $i \in\{1, \ldots, n\}$.

Proof: The fact that equations (7) and (9) are necessary follows from their derivation. To see that they are also sufficient, suppose that they were satisfied for each $i$. By contradiction, suppose that $q$ was not a central configuration. Then, for some $i$,

$$
\sum_{j \neq i}^{n} \frac{m_{j}}{r_{i j}^{\alpha+2}}\left[\begin{array}{c}
\cos \theta_{j}-\cos \theta_{i} \\
\sin \theta_{j}-\sin \theta_{i}
\end{array}\right]+\frac{\lambda}{\alpha}\left[\begin{array}{c}
\cos \theta_{i} \\
\sin \theta_{i}
\end{array}\right]=v \neq 0 .
$$

However, taking the dot product of both side of this equation with $v_{\perp}=\left[-\sin \theta_{i}, \cos \theta_{i}\right]^{T}$ and then again with $v_{r}=\left[\cos \theta_{i}, \sin \theta_{i}\right]^{T}$, we see that $v$ is orthogonal to both $v_{\perp}$ and $v_{r}$. It follows that $v=0$, and $q$ is in fact central.

\subsection{The planar $n$-vortex problem}

Many of the equations from Section 2.1 persist in the limiting case $\alpha=0$. This case corresponds to the well-known planar $n$-vortex problem, where $q_{i}$ now represents the position of the $i$-th vortex and $m_{i}=\Gamma_{i}$ is its circulation or vorticity, which may either be positive or negative. See [16] for a nice overview of the $n$-vortex problem.

For the vortex case, the analog of the potential function is actually the Hamiltonian function for the motion of the vortices. It is defined as

$$
U_{0}=-\sum_{i<j} \Gamma_{i} \Gamma_{j} \ln \left(r_{i j}\right)
$$

where $r_{i j}=\left\|q_{i}-q_{j}\right\|$ is the distance between the $i$-th and $j$-th vortices. Central configurations in the planar $n$-vortex problem are often referred to as stationary solutions or vortex crystals (see $[3,17])$. They are found by solving

$$
\sum_{j \neq i}^{n} \frac{\Gamma_{i} \Gamma_{j}\left(q_{j}-q_{i}\right)}{r_{i j}^{2}}+\omega \Gamma_{i} q_{i}=0 \quad \text { for each } i \in\{1, \ldots, n\}
$$


and for some scalar $\omega$ independent of $i$. As with the generalized Newtonian case, the system of equations defined by (10) can be written compactly as

$$
\nabla U_{0}(q)+\omega \nabla I(q)=0
$$

where $I=\frac{1}{2} \sum_{i=1}^{n} \Gamma_{i}\left\|q_{i}\right\|^{2}$ is one-half the angular impulse, but has the same meaning and definition as in Section 2.1. Taking the dot product of equation (11) with $q$ yields

$$
\omega=\frac{L}{2 I}, \quad \text { where } L=\sum_{i<j} \Gamma_{i} \Gamma_{j}
$$

is called the total angular vortex momentum.

Remark. 1. Note that the generalized Newtonian potential $U_{\alpha}$ does not limit on the vortex potential $U_{0}$ as $\alpha \rightarrow 0$. However, assuming a certain non-degeneracy condition is satisfied, it is the case that a family of central configurations for the generalized Newtonian problem will limit on a central configuration of the vortex problem. This follows using formulas (3) and (12), and observing that equation (1) transforms into equation (10) as $\alpha \rightarrow 0$.

2. Any central configuration of the planar $n$-vortex problem also begets a rigidly rotating solution (relative equilibrium). Here, $\omega \neq 0$ gives the corresponding angular velocity and since $\Gamma_{i}<0$ is allowed, we may have $\omega<0$. An example of a one-parameter family of solutions where $\omega$ flips sign occurs for a particular rhombus configuration, where two pairs of equal-strength vortices are positioned opposite each other on different axes of symmetry. If $\Gamma_{1}=\Gamma_{2}=1$ and $\Gamma_{3}=\Gamma_{4}=m$, then as $m$ decreases through $-2+\sqrt{3}$, the family passes through an equilibrium configuration $(L=0)$ and the direction of rotation of the relative equilibrium reverses (see Section 7.4 of $[9])$.

In the vortex problem, the analog of the center of mass is the center of vorticity given by $c=\frac{1}{\Gamma} \sum_{i=1}^{n} \Gamma_{i} q_{i}$, where $\Gamma=\sum_{i=1}^{n} \Gamma_{i}$ is the total circulation. A co-circular central configuration of vortices lying on the unit circle and whose center of vorticity is at the origin satisfies

$$
\sum_{j \neq i}^{n} \frac{\Gamma_{j}}{r_{i j}^{2}}\left[\begin{array}{c}
\cos \theta_{j}-\cos \theta_{i} \\
\sin \theta_{j}-\sin \theta_{i}
\end{array}\right]+\omega\left[\begin{array}{c}
\cos \theta_{i} \\
\sin \theta_{i}
\end{array}\right]=0, \quad \text { for each } i \in\{1, \ldots, n\} .
$$

As in Section 2.1, we derive two useful equations by taking the dot product of the $i$-th equation in system (13) with the vectors $\left[-\sin \theta_{i}, \cos \theta_{i}\right]^{T}$ and $\left[\cos \theta_{i}, \sin \theta_{i}\right]^{T}$, respectively. In the first case, we obtain

$$
\sum_{j \neq i}^{n} \frac{\Gamma_{j}}{r_{i j}^{2}} \sin \left(\theta_{j}-\theta_{i}\right)=0, \quad \text { for each } i \in\{1, \ldots, n\},
$$

which is precisely equation (5) with $\alpha=0$. For the second case, using identity (6), we discover the simple relation

$$
\sum_{j \neq i}^{n} \Gamma_{j}=2 \omega, \quad \text { for each } i \in\{1, \ldots, n\} .
$$


We note that equation (14) is similar to equation (16) in [4], where relative equilibria consisting of one vortex of large vorticity and $n$ identical vortices of small vorticity are studied. As shown in [4], the positions of the $n$ smaller vortices limit on a circle as their common vorticity approaches zero.

Equation (15) simplifies to $\Gamma_{i}=\Gamma-2 \omega$ for each $i$, which quickly yields the following fact.

Proposition 2.2. In the planar n-vortex problem with arbitrary vorticities, a co-circular central configuration whose center of vorticity is located at the center of the circle containing the vortices must have equal-strength circulations.

Proof: Without loss of generality, we may assume the circle containing the central configuration is the unit circle. As shown above, the circulation of the $i$-th vortex must be $\Gamma_{i}=\Gamma-2 \omega$. Since the vortices lie on the unit circle, we have that $I=\frac{1}{2} \Gamma$, and thus $\omega=L / \Gamma$. It follows that

$$
\Gamma-2 \omega=\frac{1}{\Gamma}\left(\Gamma^{2}-2 L\right)=\frac{1}{\Gamma} \sum_{i=1}^{n} \Gamma_{i}^{2} \neq 0 .
$$

Since $\Gamma-2 \omega$ is independent of $i$, the circulations must all be equal and nonzero.

\section{Main Results}

\subsection{Restricting $V_{\alpha}$ to the unit circle}

An important observation concerning equation (7) is to recognize that, for $\alpha>0$, the left-hand side is precisely $\frac{1}{\alpha m_{i}} \cdot \frac{\partial V_{\alpha}}{\partial \theta_{i}}$, where

$$
V_{\alpha}=V_{\alpha}\left(\theta_{1}, \ldots, \theta_{n}\right)=\sum_{i<j}^{n} \frac{m_{i} m_{j}}{\left[2 \sin \left(\frac{\left|\theta_{j}-\theta_{i}\right|}{2}\right)\right]^{\alpha}}
$$

is the restriction of $U_{\alpha}$ to the unit circle. For the vortex case $\alpha=0$, the key equation (14) reduces to

$$
-\frac{1}{2} \sum_{j \neq i}^{n} \frac{\delta_{i j} \Gamma_{j} \cos \left(\frac{\theta_{j}-\theta_{i}}{2}\right)}{\sin \left(\frac{\left|\theta_{j}-\theta_{i}\right|}{2}\right)}=0, \quad \text { where } \delta_{i j}= \begin{cases}1 & \text { if } \theta_{i}-\theta_{j}>0 \\ -1 & \text { if } \theta_{i}-\theta_{j}<0 .\end{cases}
$$

The left-hand side of equation (16) is equivalent to $\frac{1}{\Gamma_{i}} \cdot \frac{\partial V_{0}}{\partial \theta_{i}}$, where

$$
V_{0}=-\sum_{i<j}^{n} \Gamma_{i} \Gamma_{j} \ln \left[2 \sin \left(\frac{\left|\theta_{j}-\theta_{i}\right|}{2}\right)\right]
$$

is the restriction of $U_{0}$ to the unit circle. This shows the following lemma, one that is reminiscent of an approach introduced by Hall to study central configurations with one large body and $n$ small bodies (see [7], as well as [4, 5, 13]). 
Lemma 3.1. Fix $\alpha \geq 0$ and suppose that $q=\left(q_{1}, \ldots, q_{n}\right)$ is a central configuration on the unit circle with center of mass (or vorticity) at the origin. Then the set of angles $\theta=\left(\theta_{1}, \ldots, \theta_{n}\right)$ defining the positions of $q$ must be a critical point of $V_{\alpha}$.

Lemma 3.1 gives a particularly useful topological approach to studying solutions to equations (7) and (16). Note that if $\theta=\left(\theta_{1}, \ldots, \theta_{n}\right)$ is a critical point of $V_{\alpha}$, then so is any translation $\left(\theta_{1}+\beta, \ldots, \theta_{n}+\beta\right)$ (taken $\left.\bmod 2 \pi\right)$ for any $\beta$. This is due to the $S O(2)$ symmetry of equations (1) and (10). We can specify a unique member of this one-parameter family of critical points by requiring $\theta_{1}=0$.

Theorem 3.2. Fix $\alpha \geq 0$. Given a set of positive masses $m_{i}$ (or positive circulations $\Gamma_{i}$ if $\alpha=0$ ), for each ordering of the bodies on the unit circle, $V_{\alpha}$ has a unique critical point up to translation. This critical point is a minimum.

Proof: Our arguments follow the approach used by Moulton for collinear central configurations in the $n$-body problem $[12,15]$. Without loss of generality, suppose the bodies are arranged so that

$$
0 \leq \theta_{1}<\theta_{2}<\cdots<\theta_{n}<2 \pi
$$

On the sub-region of $[0,2 \pi]^{n}$ determined by the inequalities in (17), the function $V_{\alpha}$ is continuous, bounded below and approaches $\infty$ on the boundary. Thus, $V_{\alpha}$ attains a minimum on this region. To see that this critical point is unique up to translation, we examine the quadratic form $u^{T} D^{2} V_{\alpha}(\theta) w$, where $D^{2} V_{\alpha}$ is the Hessian matrix of $V_{\alpha}$. We compute, for $\alpha>0$, that

$$
\begin{aligned}
\frac{\partial^{2} V_{\alpha}}{\partial \theta_{i} \partial \theta_{j}} & =\frac{-\alpha m_{i} m_{j}\left(1+\alpha \cos ^{2}\left(\frac{\theta_{j}-\theta_{i}}{2}\right)\right)}{\left[2 \sin \left(\frac{\left|\theta_{j}-\theta_{i}\right|}{2}\right)\right]^{\alpha+2}} \text { if } i \neq j, \\
\frac{\partial^{2} V_{\alpha}}{\partial \theta_{i}^{2}} & =-\sum_{j \neq i}^{n} \frac{\partial^{2} V_{\alpha}}{\partial \theta_{i} \partial \theta_{j}} .
\end{aligned}
$$

The vortex problem has the same structure, but with

$$
\frac{\partial^{2} V_{0}}{\partial \theta_{i} \partial \theta_{j}}=-\frac{1}{4} \Gamma_{i} \Gamma_{j} \csc ^{2}\left(\frac{\theta_{j}-\theta_{i}}{2}\right)
$$

Suppose that $\phi=\left(\phi_{1}, \ldots, \phi_{n}\right)$ is a critical point of $V_{\alpha}$ and let $u=\left[\begin{array}{llll}u_{1} & u_{2} & \ldots & u_{n}\end{array}\right]^{T}, w=$ $\left[w_{1} w_{2} \ldots w_{n}\right]^{T}$ be arbitrary vectors. Then we have

$$
\begin{aligned}
& u^{T} D^{2} V_{\alpha}(\phi) w=\sum_{i<j}^{n} \frac{\alpha m_{i} m_{j}\left(1+\alpha \cos ^{2}\left(\frac{\phi_{j}-\phi_{i}}{2}\right)\right)}{\left[2 \sin \left(\frac{\left|\phi_{j}-\phi_{i}\right|}{2}\right)\right]^{\alpha+2}}\left(u_{i}-u_{j}\right)\left(w_{i}-w_{j}\right), \quad \text { for } \alpha>0, \\
& u^{T} D^{2} V_{0}(\phi) w=\frac{1}{4} \sum_{i<j}^{n} \Gamma_{i} \Gamma_{j} \csc ^{2}\left(\frac{\phi_{j}-\phi_{i}}{2}\right)\left(u_{i}-u_{j}\right)\left(w_{i}-w_{j}\right), \quad \text { for } \alpha=0 .
\end{aligned}
$$


Since the masses or circulations are assumed to be positive and $\left|\phi_{j}-\phi_{i}\right| / 2<\pi$ for all $i$ and $j$, it follows that $u^{T} D^{2} V_{\alpha}(\phi) u \geq 0 \forall \alpha \geq 0$, with equality if and only if $u$ is a scalar multiple of $[11 \ldots 1]^{T}$. This vector corresponds to the translational invariance of the critical point, which can be removed by specifying $\phi_{1}=0$. In this case, the quadratic form $u^{T} D^{2} V_{\alpha}(\phi) u$ is strictly positive on the tangent space so any critical point of $V_{\alpha}$ must be a minimum, and consequently, unique.

It is straight-forward to check that for any $\alpha \geq 0$, if the masses (or circulations) are identical, then the regular $n$-gon is a central configuration on the unit circle whose center of mass is at the origin. Using this fact, Theorem 3.2 has two nice corollaries with simple proofs.

Corollary 3.3. For any $\alpha>0$ and for the case of equal masses, the regular $n$-gon is the only cocircular central configuration with center of mass coinciding with the center of the circle containing the bodies.

Proof: Since the masses are all fixed, Theorem 3.2 implies that there can only be one such central configuration, and this has to be the regular $n$-gon.

\subsection{Solving the vortex case completely}

Corollary 3.4. In the planar n-vortex problem with arbitrary vorticities, the only co-circular central configuration with center of vorticity coinciding with the center of the circle is the regular n-gon with equal vorticities.

Proof: By Proposition 2.2, the circulations of the central configuration must all be equal. Without loss of generality, we can take this common circulation to be positive. By Theorem 3.2, there can only be one such possible central configuration, and this has to be the regular $n$-gon.

Remark. 1. Using a different approach, featuring a clever application of the fundamental theorem of algebra, Aref has shown that when the circulations are assumed to be equal, the only central configuration with center of vorticity coinciding with the center of the circle is the regular $n$-gon [2].

2. Corollary 3.4 contrasts with some results in the $1+n$-vortex problem $(n$ small vortices on a circle with a sufficiently large central vortex), as studied by Barry, Hall and Wayne [4]. In particular, the $1+n$-vortex problem always has at least three central configurations, although the large central vortex is not necessarily located at the origin.

Acknowledgment: The authors would like to thank the two reviewers for their helpful comments concerning this work. GR was supported by a grant from the National Science Foundation (DMS$1211675)$. 


\section{References}

[1] Albouy, A., Cabral, H. E. and Santos, A. A, Some problems on the classical $n$-body problem, Celest. Mech. Dynam. Astronom. 113 (2012), 369-375.

[2] Aref, H., Relative equilibria of point vortices and the fundamental theorem of algebra, Proc. R. Soc. A 467 (2011), 2168-2184.

[3] Aref, H., Newton, P. K., Stremler, M. A., Tokieda, T. and Vainchtein, D. L., Vortex crystals, Advances in Applied Mechanics 39 (2003), 1-79.

[4] Barry, A., Hall, G. R. and Wayne, C. E., Relative equilibria of the $(1+n)$-vortex problem, $J$. Nonlinear Sci. 22 (2012), 63-83.

[5] Casasayas, J., Llibre, J and Nunes, A., Central configurations of the planar $1+N$ body problem, Celest. Mech. Dynam. Astronom. 60 (1994), 273-288.

[6] Chenciner, A., Are there perverse choreographies? New advances in celestial mechanics and Hamiltonian systems, Kluwer/Plenum, New York (2004), 63-76.

[7] Hall, G. R., Central configurations in the planar $1+n$ body problem, preprint (1988).

[8] Hampton, M., Co-circular central configurations in the four-body problem, EQUADIFF 2003 (Conference Proceedings), World Sci. Publ., Hackensack, NJ, (2005), 993-998.

[9] Hampton, M., Roberts, G. E. and Santoprete, M., Relative equilibria in the four-vortex problem with two pairs of equal vorticities, J. Nonlinear Sci. 24 (2014), 39-92.

[10] Llibre, J. and Valls, C., The co-circular central configurations of the 5-body problem, preprint, (April, 2013).

[11] Marsden, J. E. and Ross, S. D., New methods in celestial mechanics and mission design, Bull. Amer. Math. Soc. (N.S.) 43 (2006), no. 1, 43-73.

[12] Meyer, K. R., Hall, G. R. and Offin, D., Introduction to Hamiltonian Dynamical Systems and the N-Body Problem, 2nd ed., Applied Mathematical Sciences, 90, Springer, New York, 2009.

[13] Moeckel, R., Linear stability of relative equilibria with a dominant mass, Differ. Equ. Dyn. Syst. 6 (1994), no. 1, 37-51.

[14] Moeckel, R., On central configurations, Mathematische Zeitschrift 205 (1990), no. 4, 499-517.

[15] Moulton, F. R., The straight line solutions of the problem of $n$ bodies, Ann. of Math. (2) 12 (1910), no. 1, 1-17.

[16] Newton, P. K., The N-Vortex Problem: Analytic Techniques, Springer, New York, 2001.

[17] O’Neil, K. A., Stationary configurations of point vortices, Trans. Amer. Math. Soc. 302, no. 2 (1987), 383-425. 